\title{
A Decision Fusion method to Interpret Faults using Multi-Source Remote Sensing Data
}

\author{
Xue Li, Qiuliang Wang \\ Key Laboratory of Earthquake Geodesy, Institute of \\ Seismology, CEA \\ Wuhan 430071, China
}

\author{
Zhoufeng Chen, Xin Qi, Changsheng SHAO \\ Wuhan Center of China Geological Survey \\ Wuhan 430223, China
}

\begin{abstract}
This paper summarizes the different interpretation methods using remote sensing data to detect Faults. As different data sources have respective advantages in the extraction of structural information, the paper proposes a decision fusion method to interpret faults using the ETM+ images, SPOT5 images and DEM data. The experiments show the effectiveness of the method. The research provides the basis for regional geological surveys and crustal stability assessment.
\end{abstract}

Keywords-Multi-source; Faults; Decision Fusion; Spectrum Feature; Morphological Feature

\section{INTRODUCTION}

Remote sensing technology has the characteristics of multi-temporal, multi-platform and multi-resolution. As remote sensing is suitable for large-scale observation, it is widely used in active faults detection ${ }^{[1-3]}$. With the development of remote sensing technology, the remote sensing image data obtained from different satellite sensors has become more and more rich. But the wealth of data presented a new challenge: How to make full use of multi-resource remote sensing data. Some researchers have studied the advantages of the data fusion in the extraction of geological information ${ }^{[4-6]}$. Other scholars use the middleand high-resolution remote sensing images to analyze active faults around cities ${ }^{[7-9]}$. The fusion of the optical images and radar images has been tested to obtain the information of faults ${ }^{[10]}$. On this basis, some scholars attempt to interpret the faults by optical images, radar images and GIS data ${ }^{[11,12]}$. From the previous studies we found that the effect of the interpretation using only one single data source is often not good. As we know, Multi-source data can approve the behavior. However, the interpretation method of multi-source is imperfect. Therefore, this paper proposes a method which fuses the Landsat ETM+ images, SPOT5 images and DEM data to interpret the faults.

\section{MULTI-SOURCE DATA}

In geology, a fault is a planar fracture or discontinuity in a volume of rock, across which there has been significant displacement along the fractures as a result of earth movement. A fault line is the surface trace of a fault, the line of

Corresponding author: Xue LI. E-mail:leexue1211@126.com This work is supported by the Director Foundation of the Institute of Seismology, China Earthquake Administration (IS201116002, IS201056076). intersection between the fault plane and the Earth's surface. Different fault types have different surface features and the characteristic scale. Therefore, the selection of the appropriate data sources is needed.

\section{A. Landsat ETM+ image}

Landsat ETM+ image is suitable for observation of landscape and landforms in large-scale. Because the multi-spectral bands can provide rich spectral information outside the visible light, ETM+ images have been widely used in geological survey ${ }^{[13,14]}$. Landsat 7, launched on April 15, 1999, is the latest satellite of the Landsat program. The main instrument on board Landsat 7 is the Enhanced Thematic Mapper Plus (ETM+). The ETM+ instrument has six 30-meter resolution multispectral bands (wavelength range from 0.45 to $2.35 \mu \mathrm{m})$, one 60 -meter resolution thermal infrared band (wavelength range from 10.4 to $12.5 \mu \mathrm{m}$ ) and one 15 -meter resolution panchromatic band (wavelength range from 0.52 to $0.9 \mu \mathrm{m})$.

\section{B. SPOT5 image}

Because it is suitable for obtaining land surface details, the high-resolution images have wide application in the land and resources survey. The high-resolution images used in this paper are SPOT5 images. SPOT5 is a remote sensing satellite launched by French company SPOT on May 4, 2002. The satellite sensor has three 10-meter resolution multi-spectral bands (wavelength range from 0.49 to $0.89 \mu \mathrm{m}$ ), one 20 -meter resolution thermal infrared band (wavelength range from 1.58 to $1.78 \mu \mathrm{m}$ ) and one 2.5 -meter resolution panchromatic band (wavelength range from 0.49 to $0.69 \mu \mathrm{m}$ ). SPOT5 image can be qualified for 1:250000 to $1: 10000$ scale mapping.

\section{Digital Elevation Model}

From the Digital Elevation Model (DEM), geomorphologic factors can be extracted. The geomorphologic factors, such as terrain slope and orientation, play an important role in faults interpretation. In this paper, ASTER GDEM data is used to assist faults analysis. ASTER GDEM is generated by ASTER stereo images and corrected by other data source, such as SRTM, NED, CDED and Alaska DEM. The vertical accuracy is 20 meters and the horizontal accuracy is 30 meters. 


\section{METHOD}

\section{A. Interpretation Process}

In this paper, different data source are combined to detect the faults. The interpretation process is shown in Fig. 1. First, all kinds of data are processed by the pretreatment. The pretreatment includes geometry correction, image fusion and image mosaic. Geometry correction is the process of digitally manipulating image data such that the image's projection precisely matches a specific projection surface or shape. The common methods of the geometry correction are polynomial method and collinear equation method. Image fusion can merge the high resolution panchromatic images and low resolution multi-spectral images and produces a single high resolution multi-spectral image. The common algorithms of image fusion are HIS transform, PCA transform and Wavelet transform. Image mosaic is used to splice the multiple images into a whole image. After the pretreatment, the multi-source data are interpreted respectively. And then, a preliminary interpretation result is generated by the Multi-source decision fusion. The geological data, hydrological data and other basic information are used to correct the preliminary result and the field survey is carried out to check the result.

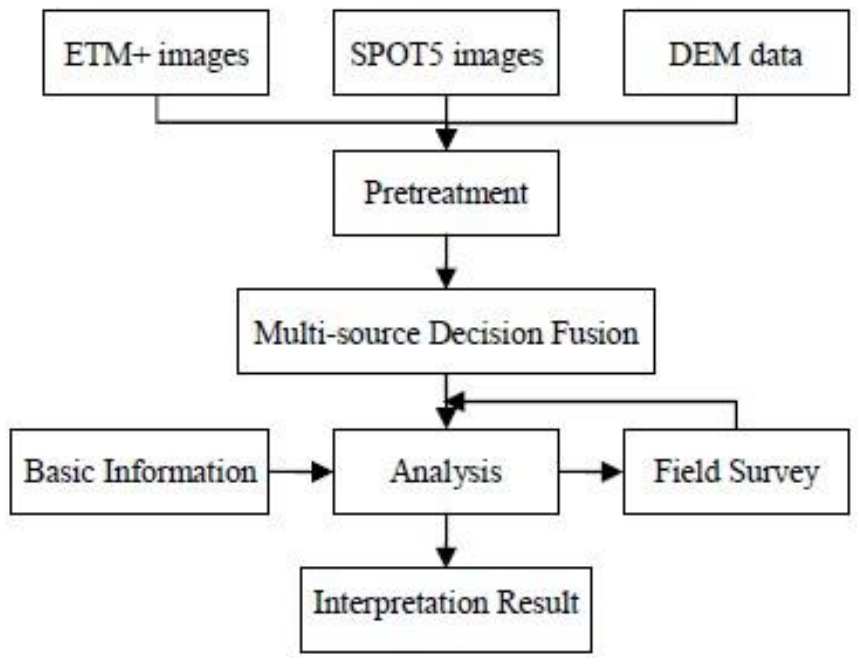

Fig.1. Faults interpretation flow chart

\section{B. Decision Fusion}

Multi-source data can approve the accuracy of the interpretation. However, different data sources increase the difficulty of the data processing. In this paper, a knowledge-based decision fusion method is used to process the multi-source data, as shown in Fig. 2. Data enhancement can help to identify the useful information. However, different source data should be enhanced separately. Because of the wealth of spectral information, spectral enhancement is suitable for the ETM+ images. False color synthetic, difference \& ratio calculation, and principal component transformation are the effective methods for spectral enhancement. For the SPOT5 images, spatial filtering, frequency domain filtering and texture enhancement are conducive to enhance the geometric characteristic of faults. DEM data can assist remote sensing image interpretation. Slope analysis, landscape rendering and $3 \mathrm{D}$ visualization make it intuitive and easy to analyze.

After the enhancement, the fault interpretation signs of different data sources can be established and the faults line can be separately extracted by the feature extraction. Then, each of the faults line is given a degree of confidence. The confidences of the faults line extracted form different data source are different with each other. For example, in the ETM+ images, the faults, which are extracted by the spectral anomalies features, may have higher degree of confidence than the faults, which are extracted by the geometric features. However, in the SPOT5 images, the situation is opposite.

Last, a weighted fusion method is used to fuse all the faults lines interpreted from different data source. The faults lines, which weights above the threshold, generate the preliminary interpretation result.

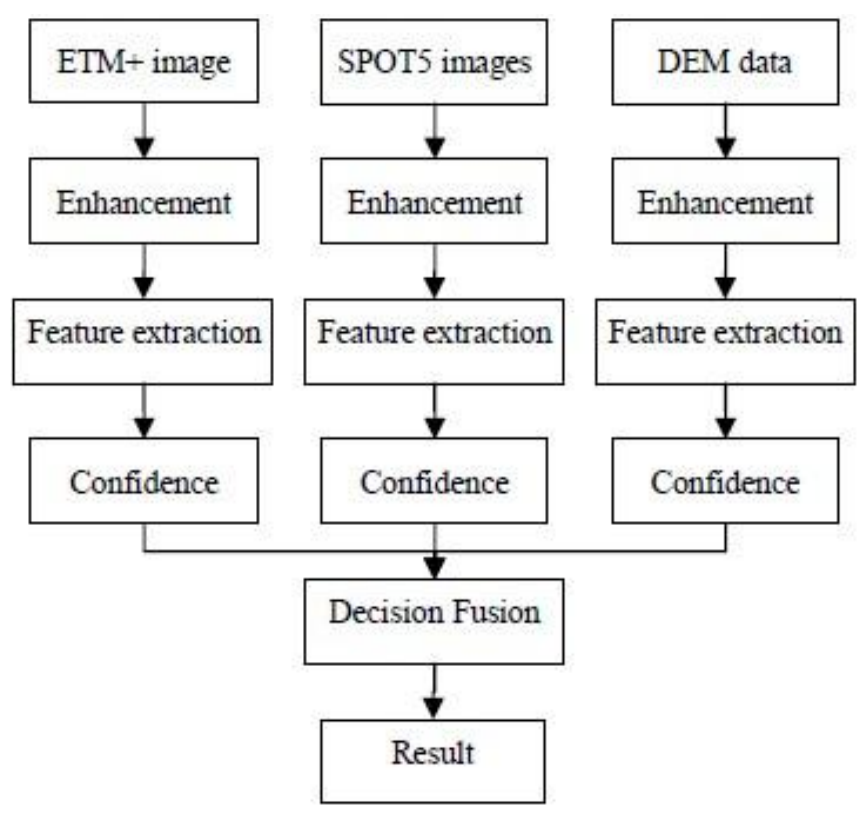

Fig.2. Multi-source data interpretation decision fusion

\section{EXPERIMENT}

An experiment is conducted to validate the proposed approach with fault interpretation using multi-source remote sensing data.

\section{A. Ribbon anomaly extraction}

Because of the differences in the types, activities and morphological characters, the spectral absorption, reflection and transmission capacity on both sides of the fault line may be different. This will cause the ribbon anomaly on the remote sensing images. The ribbon anomaly may be invisible in the visible spectrum, but it may be significant in the other bands of the spectrum. As the abundant spectral information of 
ETM+ images, it is more suitable than SPOT5 images to identify the ribbon anomaly caused by the faults. Fig. 3 shows the ribbon anomaly image and the field survey photos. Fig. 3(a) is the enhanced ETM+ image synthesized by the 7, 5, 3-band. From the image, a ribbon, which strike is NE, can be identified. The red circle on the image shows the location of the field survey, where the basalt is found on the land surface, as shown in Fig. 3(b). Fig. 3(c) shows that the color of the basalt is brown and the structure of the basalt is massive. According to the ribbon anomaly and result of survey, a NE strike buried fault can be identified.
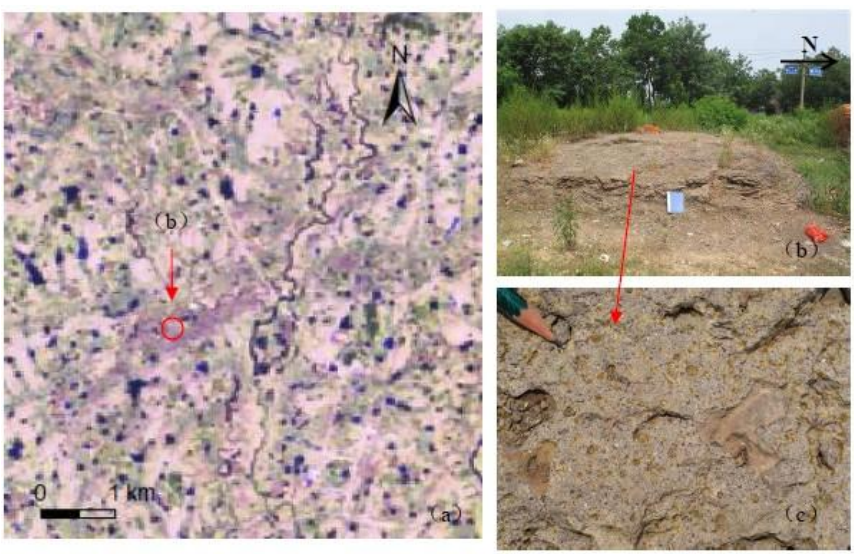

Fig.3. Ribbon anomaly image and field survey photos
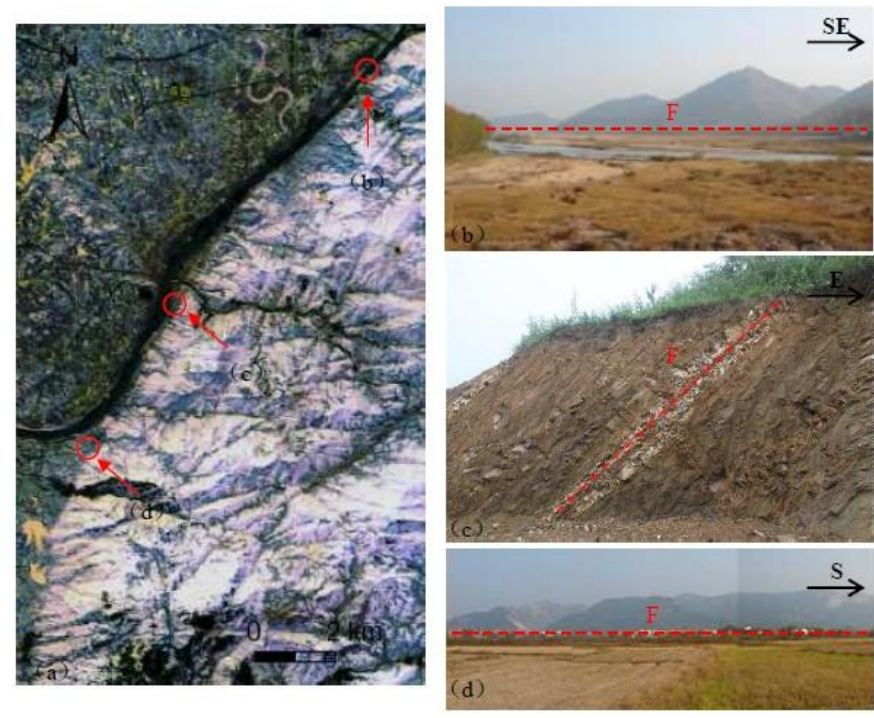

Fig.4. Linear fracture image and field survey photos

\section{B. Linear fracture extraction}

Different terrain has its own morphological characteristics. On the remote sensing image, the morphological characteristics are presented by shape, size and texture. Because of the high resolution, SPOT5 images are suitable for morphological characteristics extraction. We use SPOT5 images to extract the linear fracture, which may reveal the location of the faults. Fig. 4(a) shows the enhanced SPOT5 image. From the image, a clear line can be identified. The strike of the line is NE-NNE. Both sides of the line have different color and texture. The red circles are the location of the field survey, and the Fig.4 (b), Fig. 4 (c) and Fig. 4 (d) are the site photos. Fig. 4(b) and Fig. 4(d) show a series of fault triangles and Fig. 4(c) shows the profile of fault. The strike of the fault and fault triangles is consistent with the strike of the Lineament in Fig. 4(a).

\section{Micro-geomorphology extraction}

DEM data can assist in remote sensing interpretation. If the change of terrain is dramatic, the geomorphology information can be obtained from the remote sensing images. However, if the change of terrain is undramatic, e.g. plain, the DEM data can provide more details. Fig. 5(a) is the SPOT5 image of the plain. From the image, it is difficult to distinguish the ups and downs of the terrain. Fig. 5(b) is the $3 \mathrm{D}$ view of the same area, generated by the fusion of DEM and SPOT5. The 3D view can clearly display the change of the terrain. Fig. 5(c) and Fig. 5(d) are the profiles of the test lines marked as the black line in Fig. 5(a). Profile 1 and Profile 2 show that there is a gully on the plain. The depth of the gully is about 10 meters and the width of the gully is from hundreds meters to one kilometers. Fig. 5(e) is the photo of the field survey, which is marked as red circle in Fig. 5(a). The gully may reveal a buried fault and the result of the survey is consistent with the interpretation.

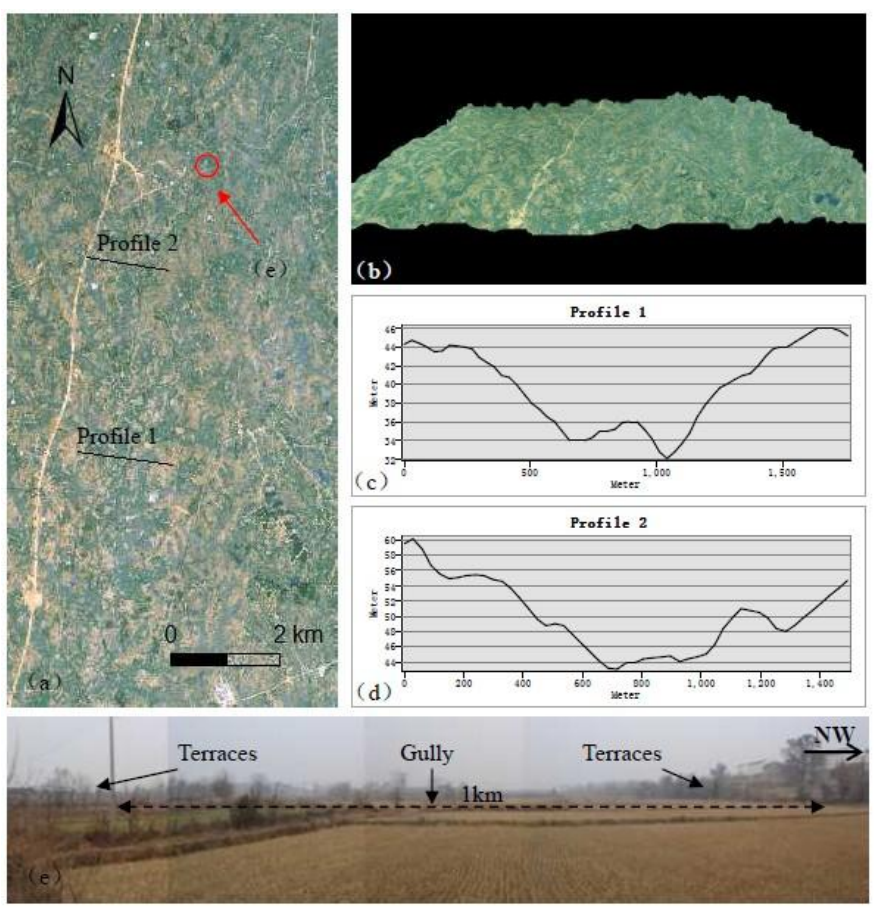

Fig.5. Micro-geomorphology extraction and field survey photo

\section{Interpretation result}

The multi-source data are interpreted separately, and the preliminary interpretation result is generated by the decision 
fusion method. Due to the complexity of the land surface, the parameters of the algorithm should be modified when the study area has changed. Therefore, the values of confidence and threshold set up to now rely on experience. After the analysis integrated with the basic information and field survey, the interpretation map is generated and shown as Fig. 6.

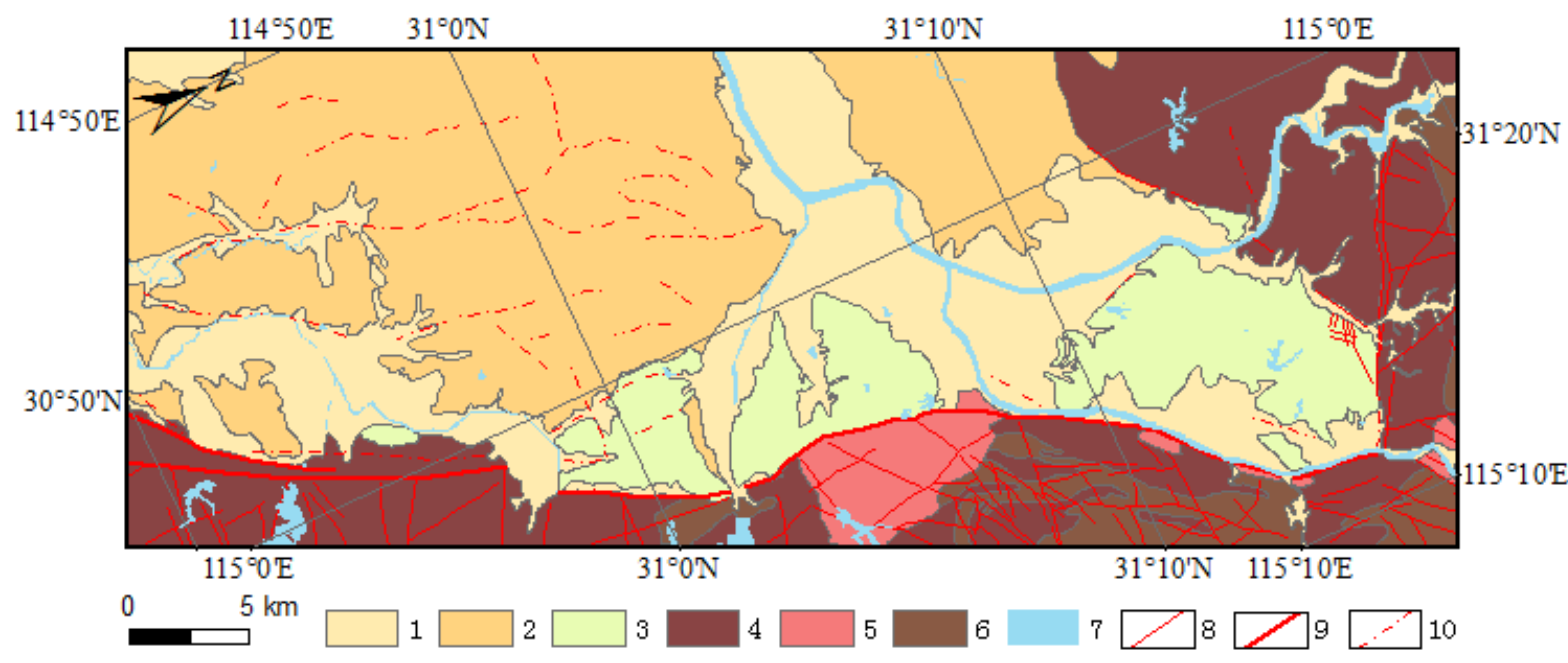

Fig.6. Interpretation map

1. Holocene 2. Pleistocene 3. Cretaceous-Paleogene 4. Anterior Cretaceous 5. Cretaceous magmatite 6. Algonkian Metamorphite 7. River \& Lake 8. Faults 9. Main Faults 10. Buried Faults

\section{V.CONCLUSIONS}

The interpretation signs of the faults are very complicated, multi-source remote sensing data can improve the accuracy of the interpretation. This paper proposes a method to make full use of the multi-source remote sensing data. The appropriate ways are used to process different data sources, and the decision fusion method is used to fuse the results interpreted by different data source. The proposed method is not perfect, however, the experiment indicates its effectiveness. The method can play a role in the applications of geological survey and crustal stability assessment.

\section{REFERENCES}

[1] Xie Guanglin. Analysis of remote sensing information on active faults in China. Beijing: Seismological Press, 2000. (in Chinese)

[2] Clark R N, et al. 2003. Imaging spectroscopy: Earth and planetary remote sensing with the USGS Tetracorder and expert systems. Journal of Geophysical Research, 108(E12): $1 \sim 44$.

[3] Zheng Wenjun, et al. Application of Remote Sensing Image Information in The Research of Active Faults. Plateau Earthquake Research, 2002, 14(2):15-21. (in Chinese)

[4] Han Ling, et al. Application of multi-homed remote sensing image data fusion method in geology. Journal of Earth Sciences and Environment, 2005,27(3):78-81. (in Chinese)

[5] Bilotti F, et al. Quantitative structural alalysis with stereocopic remote sensing imagery. AAPG Bulletin, 2000, 84(6):727 740.

[6] Clark R N, et al. Imaging Spectroscopy:Earth and Planetary Remote Sensing with the USGS Tetracorder and Expert Systems J. Geophysical Research, 2003, 108(E12):5131.

[7] Zhang Jingfa, et al. Application of remote sensing technique to exploration of active fault around urban. Journal of Natural Disaster, 2004,13(1):137-145. (in Chinese)

[8] Guo Weiying, et al. Application of Remote Sensing Technique to Active Fault Exploration in Urumqi City. Inland Earthquake, 2006, 20(1):57-64. (in Chinese)

[9] Liu Zedong, et al. Interpretation of Fault and Ground Stability Assessment Based on Remote Sensing of SPOT-5\& ETM + in Zhuhai Area. Journal of Guilin University of Technology, 2008,28(1):20-24. (in Chinese)

[10] Hong Shunying, et al. The Research on Remote Sensing Images of Nanjing Active Fault. Remote Sensing Information, 2007, 4:46-51. (in Chinese)

[11] $\mathrm{Xu}$ Yueren, et al. Identification of Major Active faults in Shenzhen Using Multi-style Remote Sensing Data. Earthquake, 2009,29:154-163. (in Chinese)

[12] Dou Aixia, et al. Remote Sensing Image Processing Technique in Active Faults Survey Based on Multi-source Data. Earthquake, 2010,30(3):123-128. (in Chinese)

[13] Han Ling, and Wang Runping. Remote Sensing Image Interpretation of Linear Tectonics in Wudu-Wenxian Area. Journal of Earth Sciences and Environment, 2008,30(4):434-437. (in Chinese)

[14] Zhang Wei, et al. The Study on Xiaoshao-Qiuchuan Faults Zone Based on Remote Sensing Technique. Bulletin of Science and Technology, 2008,24(2):156-161. (in Chinese) 